\title{
Crystallization of Dense Binary Hard-Sphere Mixtures with Marginal Size Ratio
}

\author{
Stephen R. Williams, ${ }^{1, *}$ C. Patrick Royall, ${ }^{2}$ and Gary Bryant ${ }^{3}$ \\ ${ }^{1}$ Research School of Chemistry, Australian National University, Canberra, ACT 0200, Australia \\ ${ }^{2}$ School of Chemistry, University of Bristol, Bristol, BS8 ITS, United Kingdom \\ ${ }^{3}$ Department of Applied Physics, Royal Melbourne Institute of Technology, Melbourne, Victoria 3000, Australia
}

(Received 6 March 2008; revised manuscript received 15 April 2008; published 4 June 2008)

\begin{abstract}
Molecular dynamics simulations are performed for binary hard-sphere mixtures with a size ratio of $\gamma=0.9$ and a volume fraction of $\phi=0.58$ over a range of compositions. We show how, at this high volume fraction, crystallization depends sensitively on the composition. Evidence is presented that crystallization in these mixtures does not proceed by the standard nucleation and growth paradigm. Rather, some crystallite forms almost immediately and then an interplay between compositional fluctuations and crystal growth is able to dramatically extend the time scale on which further crystallization occurs. This can be seen as a form of geometric frustration.
\end{abstract}

To be stable in the bulk amorphous solid phase, metallic glass formers must contain a mixture of several constituents [1]. The traditional explanation of the inability of a glass to crystallize is based on geometric frustration [2] which would suggest that even one component metallic glass formers should be stable, if quenched rapidly and deeply enough. The microscopic details of why metallic glass formers require several components to be stable against crystallization remain unclear. Overpacked hardsphere fluids form an important and commonly studied reference model for the glassy state [3]. For this model it is observed that a fluid of identically sized hard spheres, at high densities, crystallizes so rapidly that it is not meaningful to talk of a metastable fluid state [4]. Even randomly packed granular spheres can show signs of crystallization $[5,6]$. However, by adding a small amount of variation in the particle size (i.e., polydispersity), packing is only affected to a small degree, as are certain dynamical correlation functions measured in the overpacked fluid phase [7]. In contrast the crystallization process can be slowed down by orders of magnitude [4,7-10]. A microscopic understanding of why the crystallization of overpacked hardsphere fluids is so sensitive to the amount of polydispersity is relevant to metallic glass formers, which can be nanocrystalline [1], and is also an important problem in its own right.

Two possible resolutions of this problem have been offered. Numerical simulations combined with nucleation theory on polydisperse hard spheres have concluded that the surface tension of the nucleus rises at high volume fractions and that this effect becomes much stronger when the polydispersity is increased [11]. By contrast, MD simulations of binary hard-sphere mixtures suggest that slow compositional fluctuations are responsible for the suppression of crystallization [4]. This suggestion is supported by recent experimental studies of crystallization in hard-sphere colloids. Using skewed and symmetrical distributions [12,13], bimodal distributions [8], and the sys- tematic reduction of polydispersity through fractionation $[9,10,14]$ these studies strongly support the idea that compositional fluctuations control crystallization in polydisperse hard spheres.

We begin addressing this issue by considering the assumptions necessary for the valid application of classical nucleation theory. It is assumed that individual nuclei form spontaneously and independently from each other. Nucleation theory invokes the concept of a nucleus consisting of a bulk crystal phase, characterized by an intrinsic free energy that is less than that of the fluid, and an interface which costs free energy due to its surface tension. Thus the total free energy of the system can be reduced by decreasing the surface area of the interface or by increasing the volume of the nucleus. These effects compete against each other resulting in a critical nucleus. Any nucleus that is smaller than the critical size is thermodynamically inclined to shrink, while one that is larger is inclined to grow. These thermodynamic arguments are valid only if the critical nucleus is quite large, and are questionable for clusters with fewer than $10^{4}$ particles [15]. Although the critical nuclei of the systems we study here are much smaller than $10^{4}$, this problem can be obviated by computing the change in the free energy for the entire system upon the formation of a critical nucleus $[11,16]$. Of course one must then assume there will be a significant free energy barrier to the formation of the nucleus, a requirement which may not always be met.

Of the remaining assumptions the most relevant is that the formation of a nucleus may be realistically projected onto a single reaction coordinate. The theory can then be derived from a master equation, in the space of the reaction coordinate, with it typically being assumed that the process is in the steady state [15].

For the present case of dense binary mixtures, which form substitutional crystals $[4,17]$, we use molecular dynamics simulations to investigate the mechanism responsible for the dramatic slowing of the crystallization rate 
upon a small increase in polydispersity, and enquire whether this is compatible with nucleation theory. This is difficult, even with a large amount of computer power, because ideally it requires the simulations to have many particles, to be of a long duration, and to be repeated many times. For this reason we restricted the number of particles to a modest $N=10,976$. While it is known that many more particles are needed to completely eliminate finite size effects [18], we expect any artifacts to be quantitative only. Thus we aim to correctly identify the qualitative mechanisms, rather than to quantify a truly macroscopic system.

All simulations were of binary mixtures with a size ratio of $\gamma=\sigma_{s} / \sigma_{l}=0.9$, where the subscripts $l$ and $s$ denote the larger and smaller particles, respectively. Periodic boundary conditions with a cubic unit cell were used. The simulations, starting from a perfect FCC crystal at low volume fraction, were melted and the resulting fluid was then compressed to a volume fraction of $\phi=0.58$ (for details see [4]). The time unit is $\bar{\sigma} \sqrt{m / k_{B} T}$ where $\bar{\sigma}=$ $\left(N_{s} \sigma_{s}+N_{l} \sigma_{l}\right) / N$ is the average particle diameter. A number of compositions $X=N_{s} / N$ were simulated as given in the legend of Fig. 1. Thus the polydispersity (the standard deviation of the particle size divided by the mean particle size) varied from $s=0$ for $X=0$ (or $X=1$ ) to $s=0.054$ for $X=0.5$. Particles which are in a crystalline environment (called simply crystallite particles) were identified using the following variation on the method given in Ref. [19]. The bond network was determined using a modified Delaunay tessellation as detailed in [20]. All particles for which $\sum_{j=1}^{N_{B}(i)} \sum_{m=-6}^{6} q_{6 m}(i) q_{6 m}^{*}(j)>8.5$ were then labeled as crystallite particles, where the first sum is over all the particles bonded to the $i$ th particle, $N_{B}(i)$ is the number of particles bonded to the $i$ th particle, and the variables $q_{6 m}$ form a 13 dimensional unit vector, calculated from the 13 different $l=6$ spherical harmonics, as detailed in [19,21].

Figure 1 shows (a) the crystallinity $\left\langle p_{c}\right\rangle$ (\% of particles identified as being in a crystallite environment) and (b) the relative composition of those particles, as functions of time for various system compositions. The effect of varying the composition, or polydispersity, is readily apparent, particularly in Fig. 1(a). While the simulations which were nearly single component $(X \leq 0.3$ and $X \geq 0.825$ ) readily underwent a large degree of crystallization, the simulations with $0.5 \leq X \leq 0.78$ only crystallized a little on the time scale of the simulations. For the compositions of $X=0.5$ and $X=0.7$ the crystallization was almost totally suppressed on this time scale, with only $4.4 \%$ and $3.9 \%$ of crystallite particles, respectively. In Fig. 1(b) it can be seen that the crystallites that do form have a strongly enhanced proportion of large particles relative to the melt. Extending the simulations to $t=6500$ leads to more crystallization (7.5\% crystallite particles for $X=0.5$ and $11.5 \%$ for $X=$ $0.7)$. In slightly changing the composition from $X=0.7$ to

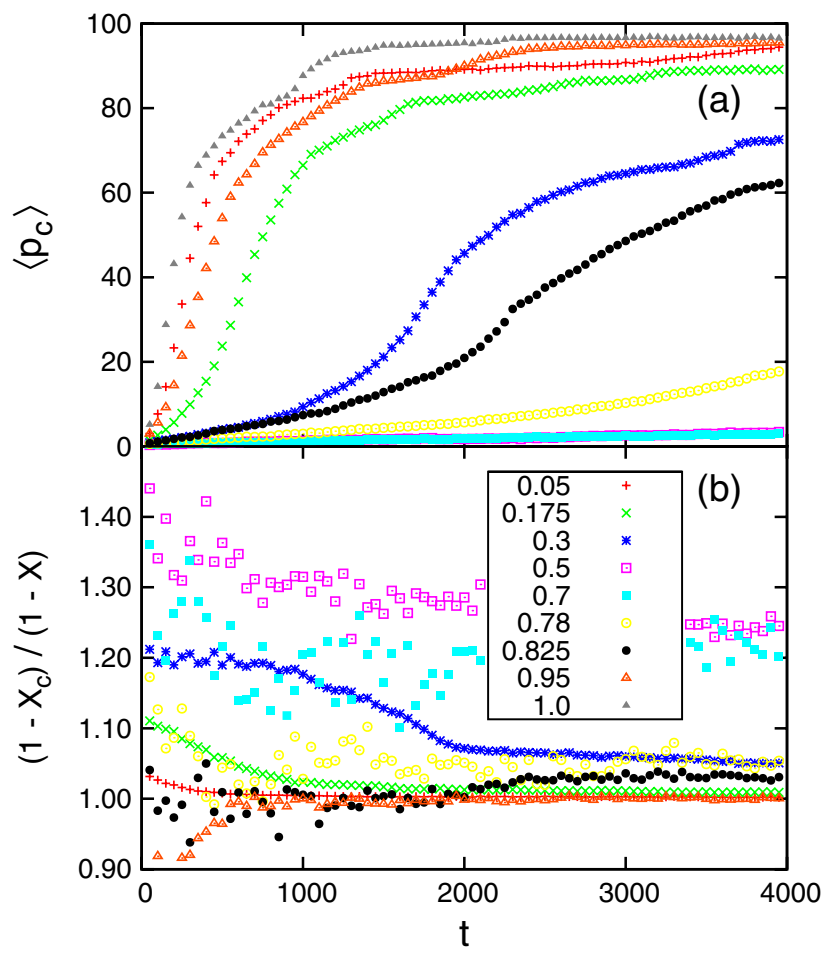

FIG. 1 (color online). Results from simulations, each repeated 10 times (except for $X=0.7$ and 0.78 which were repeated 100 times), at various compositions $X=N_{s} / N=N_{s} /\left(N_{s}+N_{l}\right)$. (a) The average percentage of particles by number identified as being crystallite $\left\langle p_{c}\right\rangle$, plotted against time $t$ for the compositions given in the legend (integer values for $N_{S}$ which gave values of $X$ closest to those in the legend were used). Note: the data for the composition of $X=0.5$ and $X=0.7$ are not distinguishably different. (b) The relative portion of large particles $\left(1-X_{c}\right) /(1-X)$ which are crystallite, where $X_{c}$ is the composition of the crystallite particles and $X$ is the composition of the total system, for compositions given in the legend (with the exception of $X=1$ ).

$X=0.78$ a considerable difference is seen in the crystallization. The composition of $X=0.78$ crystallizes with relatively fewer large particles, and does so more rapidly.

Interestingly $X=0.7$ is the composition for which the equilibrium crystal first undergoes a phase transition into two substitutional crystals at different compositions upon increasing the pressure [17,22]. In binary mixtures the eutectic composition is often associated with the suppression of freezing. While the phase diagram for this size ratio $\gamma=0.9$ is not eutectic, it does become so when it is reduced to $\gamma=0.875$ with a eutectic composition very close to $X=0.7[17,22]$.

To investigate the compositional effect on crystallization further, for $X=0.7$ we considered the largest connected region of crystallite particles for each of the 100 simulations, at the time $t=3000$ after the completion of the quench. A connected region is defined as a set of crystallite particles which are all connected such that a path exists, along the bond network for the set, between any pair of 
particles in the set. This analysis uncovered several interesting points. There were typically around $30-45$ connected regions to be found in each simulation at this time. On average, at $t=3000$, the largest connected region contained only $3 \%$ of the total particles, but $35 \%$ of the crystallite particles. This value increases to $49 \%$ at $t=$ 5000 and $60 \%$ at $t=6500$. A typical largest connected region is shown in Fig. 2 which is formed of randomly close packed plains with defects and twinning. The shape of this region is complex, containing some voids, with a very large amount of surface area relative to a sphere. This is contrary to what one would expect from thermodynamics, but for such deep overpacking, where we observe that crystallite regions can form on small length scales, an appeal to thermodynamics cannot be justified.

In Fig. 3 (main graph) a histogram of the composition of the noncrystallite particles relative to the center of mass for the largest connected region is displayed, averaged over the 100 simulations at the time of $t=3000$. This was computed by placing the periodic boundary conditions such that the connected regions center of mass corresponded with the center of the periodic cell. Close to the center of mass of the connected regions, the noncrystallite particles have an enhanced fraction of smaller particles with the larger particles incorporated into the crystallite. So we see very directly that the early stages of crystallization require a composition that is rich in large particles. For this to happen composition fluctuations, which take a long time, are required. The system does not wait indefinitely for this very slow process to occur, rather it starts to crystallizee where the composition is favorable, resulting in a nucleus with a very large surface area.

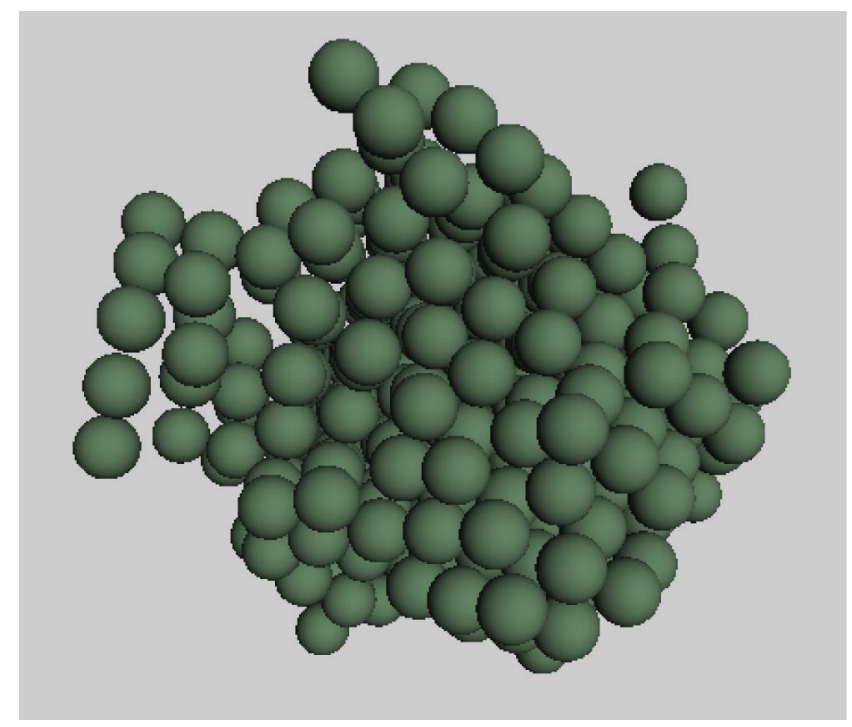

FIG. 2 (color online). A typical largest connected region of crystallite particles, taken from a simulation with $X=0.7$ at time $t=3000$. This accounts for $43 \%$ of the crystallite particles, but only $3 \%$ of the total particles.
The next question raised is the following: given that significant crystallite structure appears on such small length scales, is it long lived? To quantify this, we determined which particles were crystallite in the $X=0.7$ simulations at $t=2000$, and then determined what percentage of these particles remained crystallite at times $t=$ $2000+\tau$ for $50 \leq \tau \leq 2000$ Fig. 3 (inset). After the first 50 time units have elapsed, $\tau=50$, approximately $48 \%$ of the particles which were initially crystallite remained so. This initial rapid decay is partly due to the sensitivity of the local structure measure to small displacements, but also shows that there are some short lived structures with crystallite order. Despite this rapid initial decay, after $\tau=2000$ time units fully $35 \%$ of the particles which were initially crystallite remained so. This should be contrasted with the fact that for the whole system only $3.9 \%$ of particles are crystallite at $\tau=2000$. If the crystallite structure were not stable, the percentage of crystallite particles in the structure should decay to the overall system average. The fact that this is clearly not the case constitutes direct evidence that the system is geometrically frustrated in some manner [2], i.e., we observe locally favored structures, which are long lived, but unable to grow appreciably on the given time scale, $t<4000$.

In order to determine if the system is in a steady state in the precritical region (prior to significant crystallization), the percentage of crystallite particles $p_{c}$ was determined at different times following the quench for each of the 100 independent simulations at $X=0.7$. Figure 4 shows the probability density of finding a simulation with percentage $p_{c}$ crystallite particles at the times of $t=3000,5000$, and 6500 . The shape of the distribution, determined from the

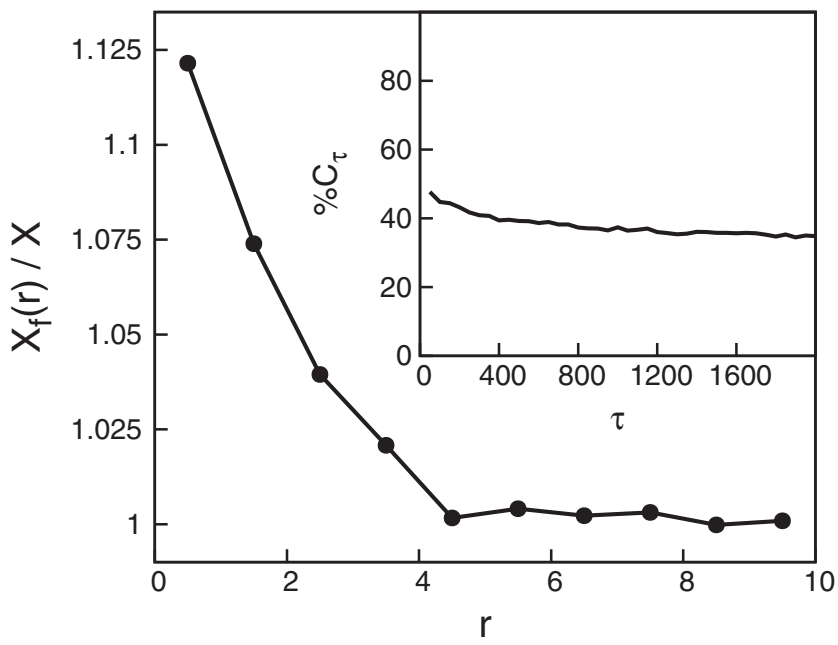

FIG. 3. Main graph: The composition of the noncrystallite particles $X_{f}(r)$, divided by the total composition $X=0.7$, as a function of the distance from the center of mass of the largest connected region of crystallite particles $r$, at the time of $t=$ 3000 after the quench. Data from 100 independent simulations. Inset: The percentage of crystallite particles, $\% C_{\tau}$, present at time $t=2000$ that remained crystallite at time $t=2000+\tau$. 


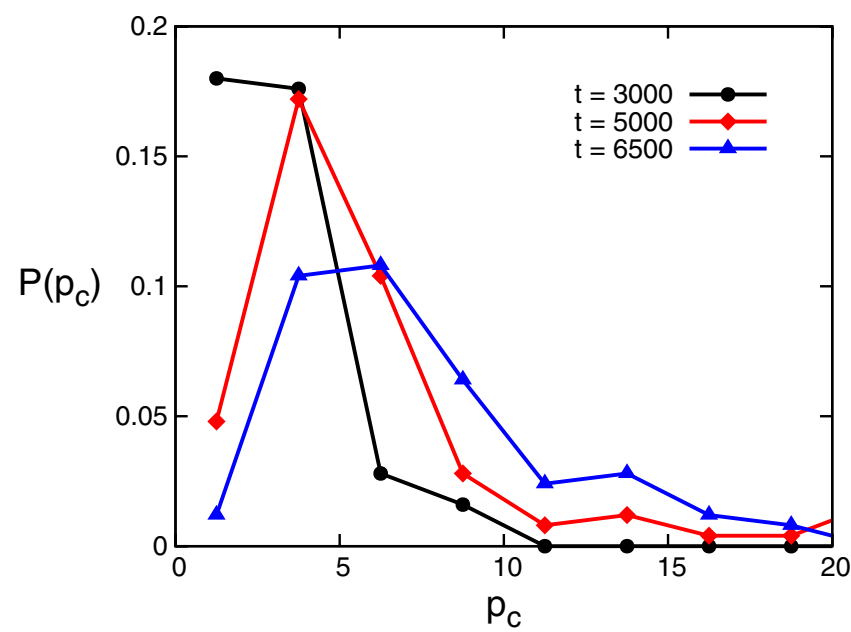

FIG. 4 (color online). The probability density of finding a simulation with percentage $p_{c}$ of crystallite particles at times of $t=3000,5000$, and 6500 , for the simulations with a total composition of $X=0.7$. The results from 100 independent simulations are shown.

ensemble of independent simulations, is clearly changing with time. At these times the average percentage of crystallite particles $p_{c}$ increases from $\left\langle p_{c}\right\rangle=3 \% \rightarrow 6 \% \rightarrow$ $11.5 \%$. For this composition the system is crystallizing in a time dependent manner and is not close to approaching the steady state. It can be seen that a limited number of the simulations undergo an appreciable amount of crystallization, breaking the geometric frustration and behaving in a manner that is in part common with the nucleation paradigm.

In summary, we have shown that the crystallization process in deeply undercooled or overpacked polydisperse fluids can be complex. For the composition of $X=0.7$ we have shown very directly that the initial crystallization process occurs by selecting regions in the fluid which have a favorable composition. Once selected, these regions remain long lived but are frustrated from further growth. These structures are consistent with precursor structures identified in both experiments $[9,10,14]$ and simulations [23]. At longer times a reasonable number of simulations were able to break this frustration and crystallization could proceed at the composition of the total system. However the system was nonergodic due to long lived crystallite structures being present and was not approaching a steady state. The frustration can be controlled by changing the composition or the size ratio. The simulations with $X=$ 0.7 showed significantly slower crystallization than did those with $X=0.78$. We would expect that reducing the size ratio below $\gamma=0.9$ would have a similar effect.

Regardless of the composition, all of the simulations were able to form a small amount of crystallite on a very short time scale, which would remain long lived and tend to grow. So strong was this tendency that it would be meaningless to consider a critical nucleus size for these systems. Either the system becomes frustrated, due to slow composition fluctuations, or it crystallizes extremely rapidly.

We thank Bill van Megen and Ian Snook for a critical reading of the manuscript and the Australian partnership for advanced computing (APAC) for computational facilities. C.P.R. acknowledges financial support from the Royal Society.

*swilliams@ @rsc.anu.edu.au

[1] A. Inoue, Acta Mater. 48, 279 (2000).

[2] D. R. Nelson and F. Spaepen, Solid State Phys. 42, 1 (1989).

[3] F. Sciortino and P. Tartaglia, Adv. Phys. 54, 471 (2005).

[4] S. R. Williams, I. K. Snook, and W. van Megen, Phys. Rev. E 64, 021506 (2001).

[5] O. Pouliquen, M. Nicolas, and P. D. Weidman, Phys. Rev. Lett. 79, 3640 (1997).

[6] T. Aste, M. Saadatfar, and T. J. Senden, Phys. Rev. E 71, 061302 (2005).

[7] S. I. Henderson, T. C. Mortensen, S. M. Underwood, and W. van Megen, Physica (Amsterdam) 233A, 102 (1996).

[8] S. Martin, G. Bryant, and W. van Megen, Phys. Rev. E 71, 021404 (2005).

[9] H. J. Schope, G. Bryant, and W. van Megen, Phys. Rev. E 74, 060401(R) (2006).

[10] H. J. Schope, G. Bryant, and W. van Megen, J. Chem. Phys. 127, 084505 (2007).

[11] S. Auer and D. Frenkel, Nature (London) 413, 711 (2001).

[12] S. Martin, G. Bryant, and W. van Megen, Phys. Rev. Lett. 90, 255702 (2003).

[13] S. Martin, G. Bryant, and W. van Megen, Phys. Rev. E 67, 061405 (2003).

[14] H. J. Schope, G. Bryant, and W. van Megen, Phys. Rev. Lett. 96, 175701 (2006).

[15] K. F. Kelton, Solid State Phys. 45, 75 (1991).

[16] S. Auer and D. Frenkel, Nature (London) 409, 1020 (2001).

[17] W. G. T. Kranendonk and D. Frenkel, Mol. Phys. 72, 679 (1991).

[18] F. H. Streitz, J. N. Glosli, and M. V. Patel, Phys. Rev. Lett. 96, 225701 (2006).

[19] P. R. ten Wolde, Ph.D. thesis, University of Amsterdam, 1998.

[20] S. R. Williams, arXiv:0705.0203.

[21] P. J. Steinhardt, D. R. Nelson, and M. Ronchetti, Phys. Rev. B 28, 784 (1983).

[22] The compositon $X$ as reported in [17] corresponds to $1-X$ here.

[23] B. O’Malley and I. Snook, J. Chem. Phys. 123, 054511 (2005). 